\title{
Application of the revised Tumour Node Metastasis (TNM) staging system of clear cell renal cell carcinoma in eastern China: advantages and limitations
}

\author{
Chao Qin ${ }^{1 *}$, Li-Jiang Sun ${ }^{2 *}$, Li Cui ${ }^{3}$, Qiang Cao ${ }^{1}$, Jian $\mathrm{Zhu}^{1}, \mathrm{Pu} \mathrm{Li}^{1}$, Gui-Ming Zhang ${ }^{2}$, Xin Mao ${ }^{2}$, Peng-Fei Shao ${ }^{1}$, \\ Mei-Lin Wang ${ }^{4}$, Zheng-Dong Zhang ${ }^{4}$, Min Gu ${ }^{1}$, Wei Zhang ${ }^{1}$ and Chang-Jun Yin ${ }^{1}$
}

This study was designed to evaluate whether the revised 2010 Tumour Node Metastasis (TNM) staging system could lead to a more accurate prediction of the prognosis of renal cell carcinoma (RCC) patients. A total of 1216 patients who had undergone radical nephrectomy or partial nephrectomy for RCC from 2003 to 2011 were enrolled. All of the patients had pathologically confirmed clear cell RCC (ccRCC). All cases were staged by both the 2002 and 2010 TNM staging systems after pathological review, and survival data were collected. Univariate and multivariate Cox regression models were used to evaluate cancer-specific survival (CSS) and progression-free survival (PFS) after surgery. Continuous variables, such as age and tumour diameter, were calculated as mean values and standard deviations (s.d.) or as median values. Survival was calculated by the Kaplan-Meier method, and the log-rank test assessed differences between groups. Statistically significant differences in CSS and PFS were noted among patients in T3 subgroups using the new 2010 staging system. Therefore, the revised 2010 TNM staging system can lead to a more accurate prediction of the prognosis of ccRCC patients. However, when using the revised 2010 staging system, we found that more than $92 \%$ of patients (288/313) with T3 tumours were staged in the T3a subgroup, and their survival data were not significantly different from those of patients with T2b tumours. In addition, T2 subclassification failed to independently predict survival in RCC patients.

Asian Journal of Andrology (2013) 15, 550-557; doi:10.1038/aja.2012.161; published online 8 April 2013

Keywords: kidney neoplasm; prognosis; renal cell carcinoma; TNM stage

\section{INTRODUCTION}

Renal cell carcinoma (RCC) is the most common malignancy of the kidney, and its occurrence is increasing in both men and women. RCC is found worldwide, with its highest incidence in developed countries. ${ }^{1}$ It is estimated that approximately 37.7 men and 16.6 women per 100000 Chinese people are diagnosed with RCC every year. ${ }^{2}$

The Tumour Node Metastasis (TNM) staging system is widely used to define the anatomic extent of the disease, to indicate the prognosis, to classify the tumour for a treatment plan, to evaluate the results of different studies and to facilitate the exchange of information. An older (sixth edition) TNM staging system for RCC, from 2002, has been described previously. ${ }^{3}$ With the revision of its pathological features in 2010 (seventh edition), stage T2 in the new staging system was divided into two subgroups according to the tumour size (T2a $>7 \mathrm{~cm}$ and $\leqslant 10 \mathrm{~cm}$; T2b $>10 \mathrm{~cm}$ ). Furthermore, the new staging system directly reclassified the adrenal gland invasion from T3a to T4, and the renal vein invasion from T3b to T3a. These changes were evidenced by the fact that the reassignment of pathological features to specific stages could more accurately predict the cancer-specific survival (CSS) and progression-free survival (PFS) associated with an increased $\mathrm{T}$ stage. $^{4,5}$

Using the 2002 and 2010 TNM staging systems, this retrospective study aimed to compare the accuracy of prognosis prediction in 1216 patients who underwent radical nephrectomy or partial nephrectomy for RCC from 2003 to 2011. All patients were pathologically confirmed to have clear cell RCC (ccRCC).

\section{MATERIALS AND METHODS}

Subjects/patients

A total of 1216 patients with ccRCC confirmed by pathological examination were enrolled from 2003 to 2011 in three hospitals (Department of Urology in the First Affiliated Hospital of Nanjing Medical University, Nanjing, China; the Affiliated Hospital of Medical College Qingdao University, Qingdao, China; and the Third Affiliated Hospital of Soochow University, Changzhou, China). Patients' information, such as age and sex, and tumour characteristics, such as tumour

${ }^{1}$ Department of Urology, The First Affiliated Hospital of Nanjing Medical University, Nanjing 210029, China; ${ }^{2}$ Department of Urology, The Affiliated Hospital of Medical College Qingdao University, Qingdao 266001, China; ${ }^{3}$ Department of Urology, The Third Affiliated Hospital of Soochow University, Changzhou 213003 , China and ${ }^{4}$ Department of Molecular and Genetic Toxicology, School of Public Health, Nanjing Medical University, Nanjing 210029, China

* These authors contributed equally to this work.

Correspondence: Dr CJ Yin (drcjyin@gmail.com) and DrW Zhang (doctormn@163.com)

Received: 27 September 2012; Revised: 24 November 2012; Accepted: 12 December 2012; Published online: 8 April 2013 
diameter, stage and Fuhrman grade, were obtained from the hospital databases. We recorded the tumour stage according to the 2002 staging system as originally documented in the reports and then reassigned the tumour stage according to the revised 2010 staging system.

\section{Follow-up and evaluation}

The duration of follow-up was calculated from the date of the operation to the date of death or last follow-up. Exact data, such as the causes of death of each patient, were acquired from the patients' family members or hospital records if the patient had been followed up or had died. During follow-up, patients were reviewed every 6 months with clinical and radiological assessments such as chest and abdomen computed tomography scan when necessary. The last follow-up occurred during December 2011, during which the CSS and PFS were measured. The Response Evaluation Criteria in Solid Tumours evaluation standard was introduced to analyse PFS. In cases with target metastatic lesions, the progression of disease was evaluated when the maximum diameter of the target lesion increased to $20 \%$ greater than that of the smallest lesion recorded after the beginning of treatment or when one or more new lesions developed. In cases with non-target metastatic lesions, progression of disease was evaluated when one or more new lesions developed and/or when the non-target lesions showed definite progress.

\section{Statistical analysis}

Continuous variables, such as age and tumour diameter, were calculated as mean \pm standard deviation (s.d.) and were compared by $t$-test. Differences in survival times according to disease stage were estimated using the Kaplan-Meier method and were compared using the logrank test. $P<0.05$ was considered statistically significant, and all statistical tests were two-sided. All of the statistical analyses were performed with SPSS 17.0 (SPSS Inc, Chicago, IL, USA).

\section{RESULTS}

The patients' clinical and pathological characteristics are listed in Table 1. The mean age of the patients was $57.5 \pm 11.5$ years. In total, 794 patients $(65.3 \%)$ were male, and 422 patients $(34.7 \%)$ were female. The mean tumour size of all patients was $6.2 \pm 3.7 \mathrm{~cm}$. At a median follow-up of 35.0 months, 96 (7.9\%) patients had died of the disease, while 119 (9.8\%) patients demonstrated disease progression.

The $\mathrm{N}$ and $\mathrm{M}$ stagings did not change substantially between the 2002 and 2010 systems, so we compared the $\mathrm{T}$ stage distribution according to the 2002 and 2010 TNM staging systems (Table 1). Based on the 2002 staging system, there were 503,173, 178, 114, 203, 13 and 32 patients in stages T1a, T1b, T2, T3a, T3b, T3c and T4, respectively. Kaplan-Meier curves showed that the cumulative CSS and PFS were statistically significantly different between consecutive increases in $\mathrm{T}$ stage (both $P<0.001$, Figure $\mathbf{1 a}$ and $\mathbf{1 b}$ ). We then reclassified tumour $\mathrm{T}$ stage according to the revised $2010 \mathrm{~T}$ staging system. The number of patients in stages T1 and T2 remained the same, but the T2 stage was divided into two subgroups, with 116 and 62 patients in stages T2a and $\mathrm{T} 2 \mathrm{~b}$, respectively. We noted an increase in the number of patients in stage T3a, from 114 to 288 , and a decrease in the number of patients in stage T3b, from 203 to 12 , because patients with renal vein invasions were reclassified; the number of patients in stage $\mathrm{T} 3 \mathrm{c}$ remained the same. We also noted that 17 patients were reclassified to stage T4 according to the new staging system because of adrenal gland invasion. After reclassification, we found that the cumulative CSS and PFS demonstrated statistically significant, measurable differences between
Table 1 Clinical and pathologic features of the 1216 analysed patients with ccRCC

\begin{tabular}{lc}
\hline Variables & \\
\hline Age (year) (mean \pm s.d.) & $57.5 \pm 11.5$ \\
Sex & \\
Male, $n$ (\%) & $794(65.3)$ \\
Female, $n$ (\%) & $422(34.7)$ \\
Pathologic tumour size (cm) (mean \pm s.d.) & $6.2 \pm 3.7$ \\
Pathological T stage (TNM 2002), $n$ (\%) & \\
T1a & $503(41.4)$ \\
T1b & $173(14.2)$ \\
T2 & $178(14.6)$ \\
T3a & $114(9.4)$ \\
T3b & $203(16.7)$ \\
T3c & $13(1.1)$ \\
T4 & $32(2.6)$ \\
Pathological T stage (TNM 2010), $n$ (\%) & \\
T1a & $503(41.4)$ \\
T1b & $173(14.2)$ \\
T2a & $116(9.5)$ \\
T2b & $62(5.1)$ \\
T3a & $288(23.7)$ \\
T3b & $12(1.0)$ \\
T3c & $13(1.1)$ \\
T4 & $49(4.0)$ \\
N staging, $n$ (\%) & \\
Negative & $1187(97.6)$ \\
Positive & $29(2.4)$ \\
M staging, $n$ (\%) & \\
Negative & $1187(97.6)$ \\
Positive & $29(2.4)$ \\
Clinical stage, $n$ (\%) & \\
I & \\
II & $645(53.0)$ \\
III & $150(12.3)$ \\
IV & $329(27.1)$ \\
I & $92(7.6)$ \\
II & \\
III & \\
IV & $305(25.1)$ \\
\hline
\end{tabular}

Abbreviations: ccRCC, clear cell renal cell carcinoma; s.d., standard deviation TNM, Tumour Node Metastasis.

consecutive increases in $\mathrm{T}$ stage according to the 2010 staging system (both $P<0.001$, Figure 1c and 1d).

As shown in Table 2, $\mathrm{T}$ stage, tumour grade, and metastasis status were correlated with CSS in the univariate analysis. However, in the multivariate analysis of potential prognostic factors for patients with ccRCC, only $\mathrm{T}$ stage and metastasis status could be confirmed as significant independent prognostic factors correlated with CSS. Similar results were noted in the univariate and multivariate analyses for PFS (data not shown).

According to the 2002 staging system, we found that the prognosis of patients with T3b tumours was better than that of patients with T3a tumours, as the cumulative CSS was statistically significantly different between these two groups $(P<0.001$, Figure 2a). Although the differences in cumulative PFS were not significantly different, there was still a similar trend between these two groups $(P=0.065$, Figure $2 \mathbf{b})$. However, when we re-analysed these data by using the 2010 staging system, we found that the prognosis of patients with $\mathrm{T} 3 \mathrm{~b}$ tumours was worse than that of patients with T3a tumours; both the cumulative 
Table 2 Univariate and multivariate analyses of patient and tumour characteristics with regard to their prognostic impact on cancer-specific survival (Cox regression analysis)

\begin{tabular}{|c|c|c|c|c|}
\hline Variables & \multicolumn{2}{|c|}{ Univariate analysis } & \multicolumn{2}{|c|}{ Multivariate analysis } \\
\hline Age & $1.00(0.98-1.02)$ & 0.827 & $0.99(0.98-1.01)$ & 0.702 \\
\hline Female & 1.00 (reference) & & 1.00 (reference) & \\
\hline Male & $1.69(1.06-2.68)$ & 0.027 & $1.15(0.76-1.74)$ & 0.401 \\
\hline \multicolumn{5}{|l|}{ Pathological T stage } \\
\hline T2a & $1.40(0.27-7.27)$ & 0.692 & $4.50(0.37-54.4)$ & 0.864 \\
\hline $\mathrm{T} 2 \mathrm{~b}$ & $2.60(0.61-11.09)$ & 0.196 & $8.12(0.76-87.02)$ & 0.650 \\
\hline ТЗа & 5.85 (2.29-14.93) & $<0.001$ & $6.84(0.75-62.34)$ & $<0.001$ \\
\hline T3b & $35.11(10.14-121.52)$ & $<0.001$ & $24.49(2.18-275.19)$ & $<0.001$ \\
\hline T3c & $46.64(14.64-148.65)$ & $<0.001$ & $38.18(3.926-371.30)$ & $<0.001$ \\
\hline $\mathrm{T} 4$ & $79.70(30.92-205.47)$ & $<0.001$ & $82.98(9.11-756.33)$ & $<0.001$ \\
\hline IV & $5.72(2.87-11.42)$ & $<0.001$ & $1.01(0.50-2.02)$ & 0.174 \\
\hline \multicolumn{5}{|l|}{ Metastasis status } \\
\hline Negative $\left(\mathrm{N}_{0}\right.$ and $\left.\mathrm{M}_{0}\right)$ & 1.00 (reference) & & 1.00 (reference) & \\
\hline Positive $(\mathrm{N}+$ and/or $\mathrm{M}+$ ) & $11.81(7.18-19.43)$ & $<0.001$ & $3.96(2.24-6.99)$ & $<0.001$ \\
\hline
\end{tabular}

Abbreviations: $\mathrm{Cl}$, confidence interval; $\mathrm{HR}$, hazard ratio.

CSS and PFS were statistically significantly different between these two groups (both $P<0.001$, Figure $2 \mathrm{c}$ and $2 \mathrm{~d}$ ).

In the 2010 TNM staging system, patients with renal vein invasions were reclassified as T3a, which resulted in 201 patients being downstaged to T3a. The Kaplan-Meier analysis showed that patients whose disease was downstaged had significantly better survival than those who remained classified as T3b $(P<0.001$ for CSS, Figure 3a; $P=0.001$ for PFS, Figure $3 \mathbf{b}$ ), indicating that this reclassification better demonstrates the survival of patients with diverse outcomes. Further, 17 patients were shifted from T3a to T4 because adrenal gland invasion was reclassified into stage T4. Similarly, the Kaplan-Meier analysis showed that patients reclassified as T4 had poorer survival than patients whose T3a classification did not change $(P<0.001$ for CSS, Figure 3c; $P<0.001$ for PFS, Figure 3d).

According to the 2010 new T staging system, the T2 group was divided into two subgroups, with 116 patients in stage T2a and 62 patients in T2b. Applying the Kaplan-Meier analysis to compare these two subgroups, we found no significant differences in CSS and PFS between the T2a and T2b subgroups ( $P=0.364$ for CSS, Figure 4a; $P=0.093$ for PFS, Figure $4 \mathbf{b})$. Furthermore, when we excluded patients with lymph node or distant metastases, we found that there was no significant difference in CSS or PFS between these two subgroups $(P=0.900$ for CSS, Figure $4 \mathrm{c} ; P=0.251$ for PFS, Figure 4d).

Provided that the major revision in the 2010 TNM staging system was in the T3 staging, we focused on the effect of this update on survival outcomes. According to the 2002 staging system, there were 114, 203 and 13 patients in stages T3a, 3b and 3c, respectively. When reclassified by the 2010 version, we noticed that most of patients with T3 tumours were grouped into the T3a stage (92.01\%), while the Kaplan-Meier analysis did not show that patients with T3a tumours had poorer survival than those with T2b tumours $(P=0.203$ for CSS, Figure $5 \mathbf{a} ; P=0.604$ for PFS, Figure $5 \mathbf{b})$. Therefore, we considered whether it was necessary to divide the T3a stage into several subgroups to more accurately predict the prognosis. To validate this hypothesis, we divided the T3a group into subgroups. First, we used a 7-cm cutoff to analyse PFS and CSS and found that the results did not show any significant difference unless the cutoff was increased to $10 \mathrm{~cm}$. Thus, we divided the T3a group into two subgroups according to the diameter of the tumour (subgroup $1 \leqslant 10 \mathrm{~cm}$, subgroup $>10 \mathrm{~cm}$ ), and the Kaplan-Meier analysis showed that patients with subgroup2 tumours had poorer survival rates than $\mathrm{T} 2 \mathrm{~b}$ and subgroup 1 patients (CSS rate $=0.040$ and 0.017 for subgroup 2 vs. T2b and subgroup1, respectively, Figure 5c; PFS rate $=0.222$ and 0.0478 for subgroup2 $v s$. T2b and subgroup1, respectively, Figure 5d). However, the survival data were not significantly different between subgroup 1 and T2b patients (CSS and PFS rate $=0.582$ and 0.681 , respectively, Figure 5c and 5d). Second, we divided the T3a group into three subgroups according to the extension of tumour invasion (tumour invading the perirenal and/or renal sinus fat as subgroup1, tumour grossly invading the renal vein as subgroup2, and tumour invading the perirenal and/or renal sinus fat and renal vein as subgroup3) and found that patients with subgroup3 tumours had poorer survival than patients in subgroup1 and subgroup2 (all $P<0.001$ for CSS and PFS, Figure $5 \mathbf{e}$ and $\mathbf{5 f}$ ). However, the survival data were not significantly different between subgroup1 and subgroup2 (Figure 5e and 5f). Furthermore, we found that patients with subgroup3 tumours had better survival than those in the T3b, T3c or T4 groups $(P=0.016$ for PFS, $P=0.079$ for CSS) (Figure 6a and 5b).

\section{DISCUSSION}

Most researchers believe that the difference in the incidence of ccRCC between developed countries and developing countries might be based on different levels of diagnosis, autopsy rates and environmental factors. The influence of the patient's ethnic background on RCC is not as evident as in other tumours, such as prostate cancer. Thus, the TNM 

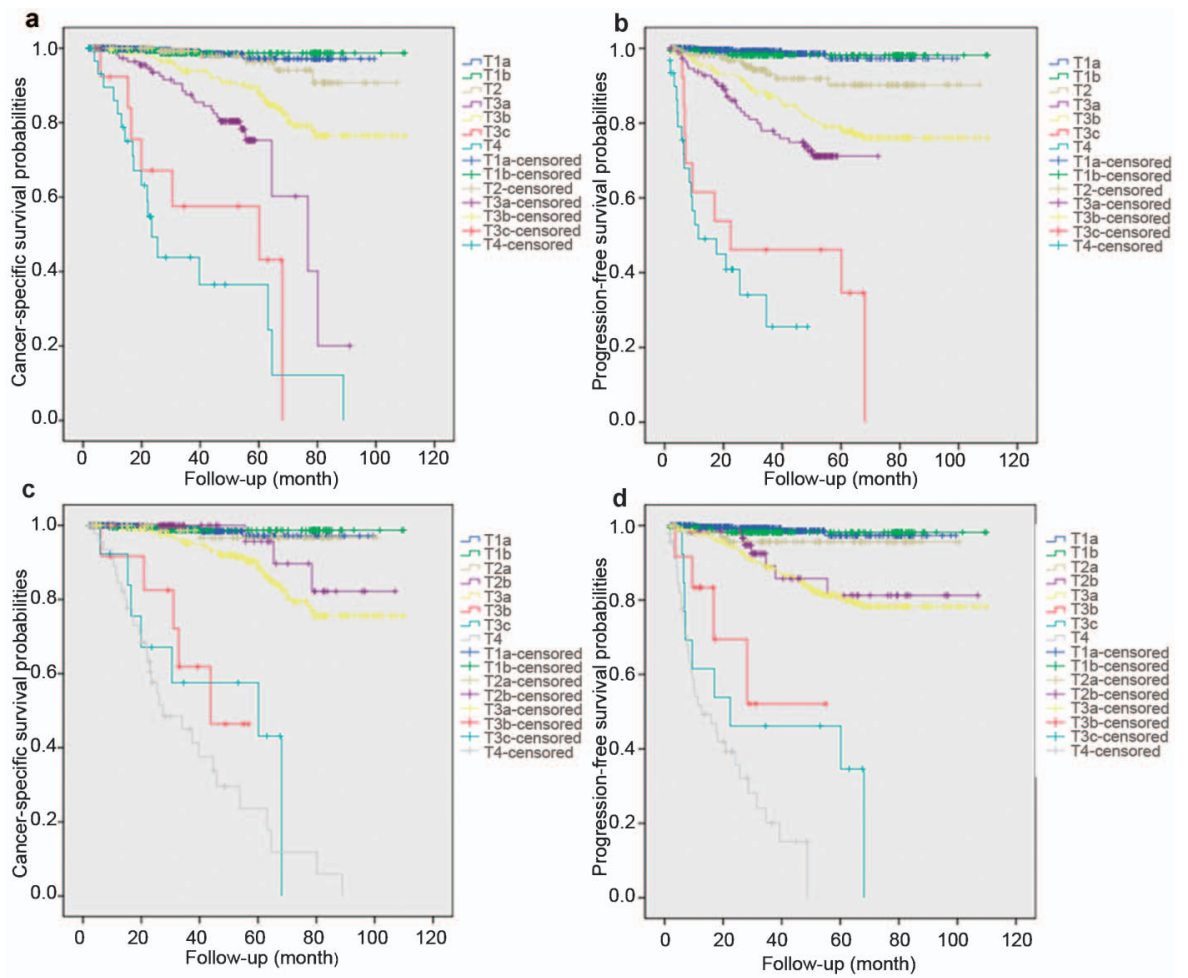

Figure 1 These Kaplan-Meier curves show that the cumulative CSS and PFS were significantly different between consecutive increases in T stage, according to the 2002 (a, b) and the 2010 (c, d) classification systems. CSS, cancer-specific survival; PFS, progression-free survival.
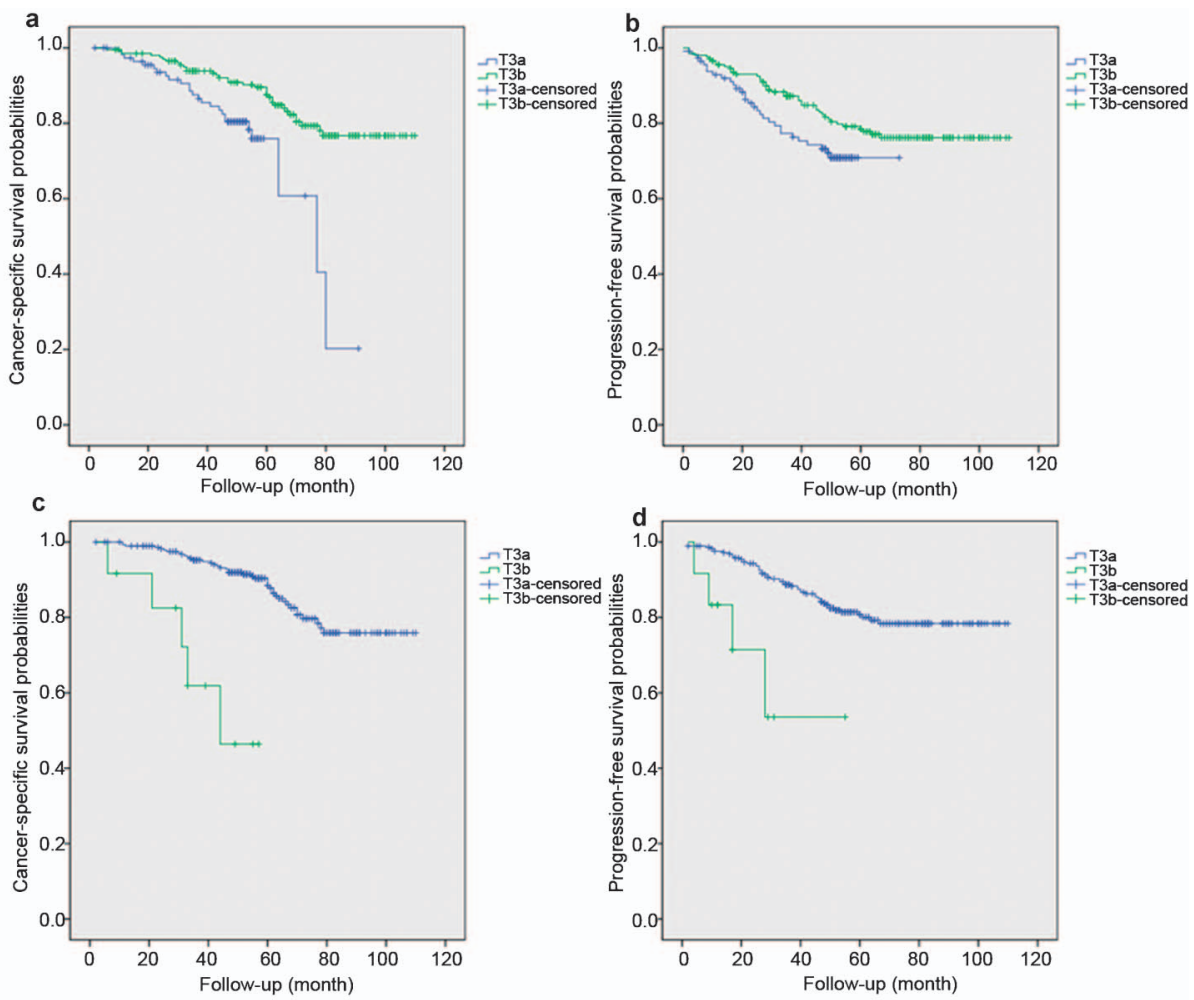

Figure 2 These Kaplan-Meier curves show that the prognosis of patients with T3b was better than that of patients with T3a tumours according to the 2002 staging system (a: $P<0.001$ for CSS; $\mathbf{b}: P=0.065$ for PFS). In the 2010 staging system, the prognosis of patients with T3b was worse than that of patients with T3a tumours (c: $P<0.001$ for CSS; $\mathbf{d}: P<0.001$ for PFS). CSS, cancer-specific survival; PFS, progression-free survival. 

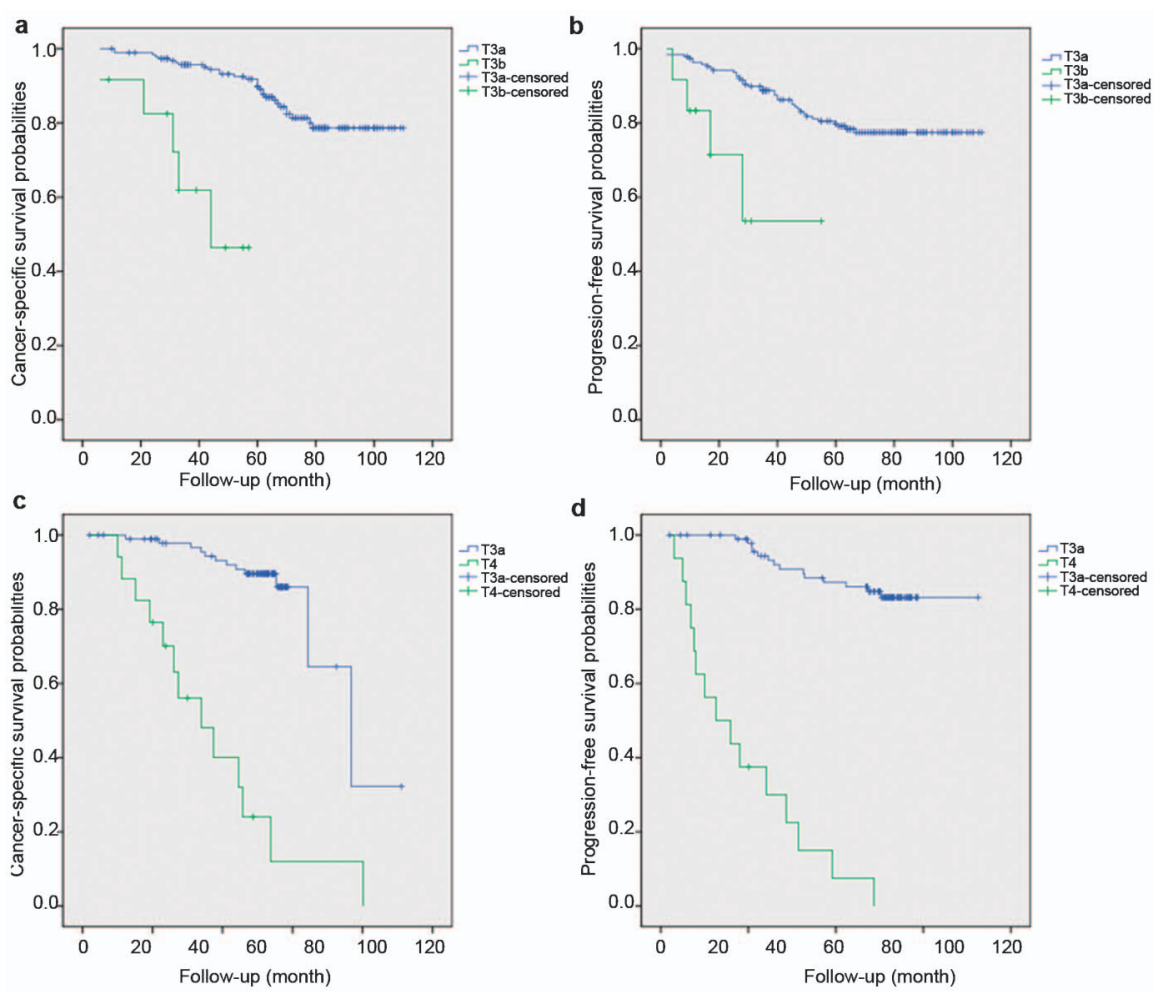

Figure 3 Survival curves of patients with diseases downstaged from T3b to T3a and of those with diseases remaining in T3b ( $a$ : $P<0.001$ for CSS; $\mathbf{b}$ : $P=0.001$ for $P F S$ ). Survival curves of patients with diseases upstaged from T3a to T4 and of those with disease remaining in T3a (c: $P<0.001$ for CSS; $\mathbf{d}: P<0.001$ for PFS). CSS, cancerspecific survival; PFS, progression-free survival.
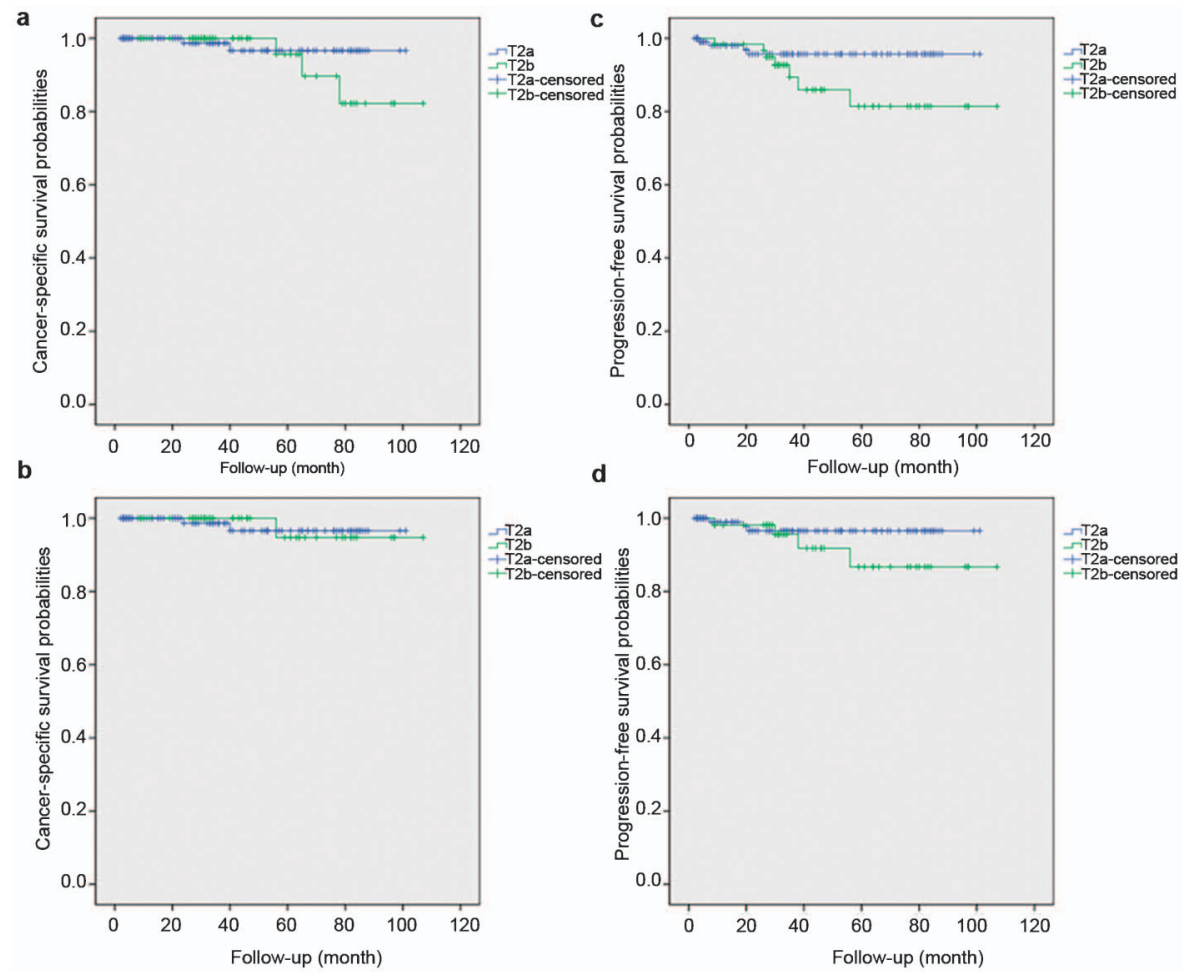

Figure 4 These Kaplan-Meier curves show that the CSS and PFS rates for the T2a and T2b subgroups were not significantly different (a: $P=0.364$ for CSS; $\mathbf{b}$ : $P=0.093$ for PFS). For the subgroup of patients without lymph node or distant metastasis $\left(N_{0} / M_{0}\right)$, there was still no significant difference between the CSS and PFS rates of these two subgroups (c: $P=0.900$ for CSS; $\mathbf{d}$ : $P=0.251$ for PFS). CSS, cancer-specific survival; PFS, progression-free survival. 

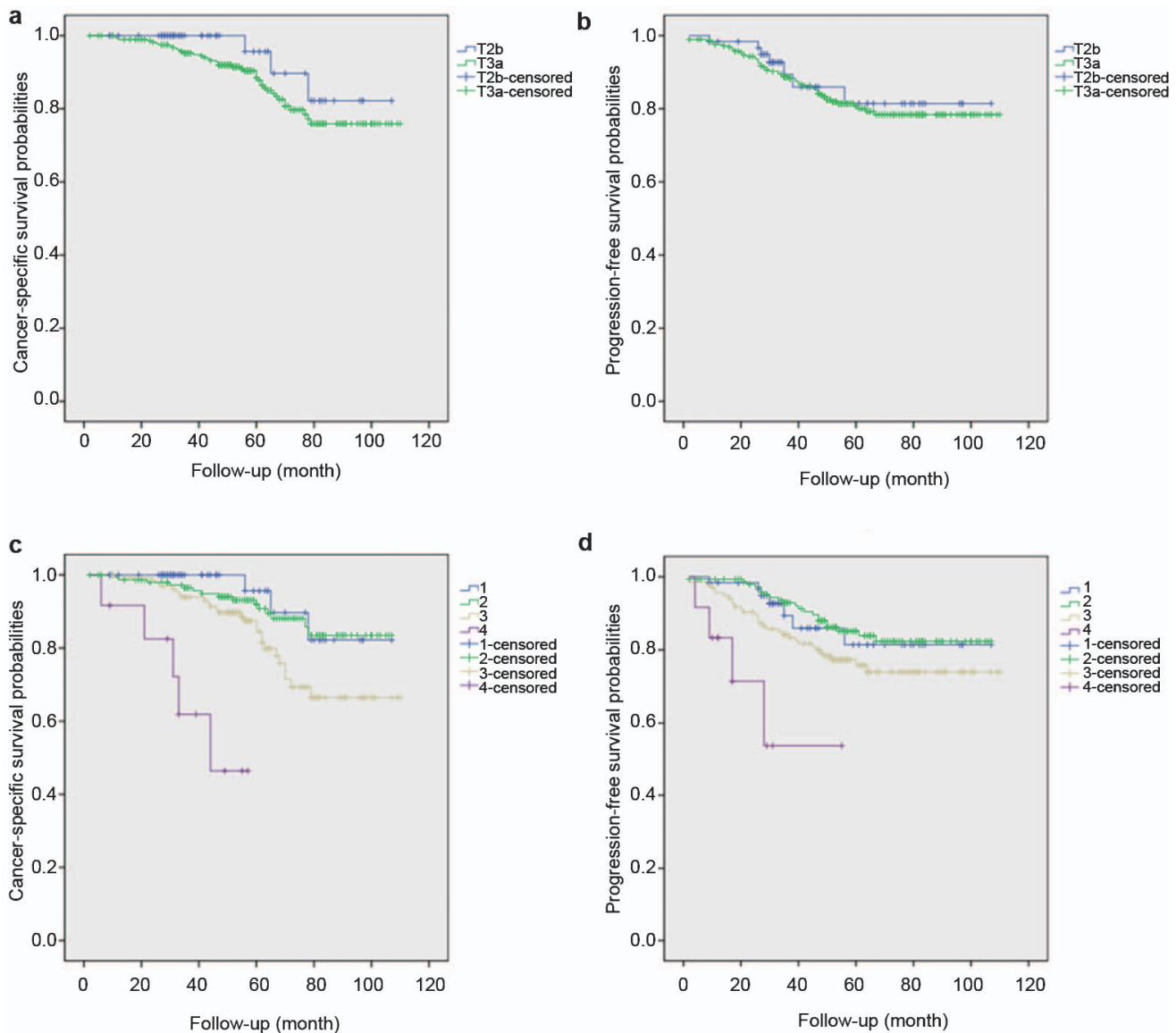

d
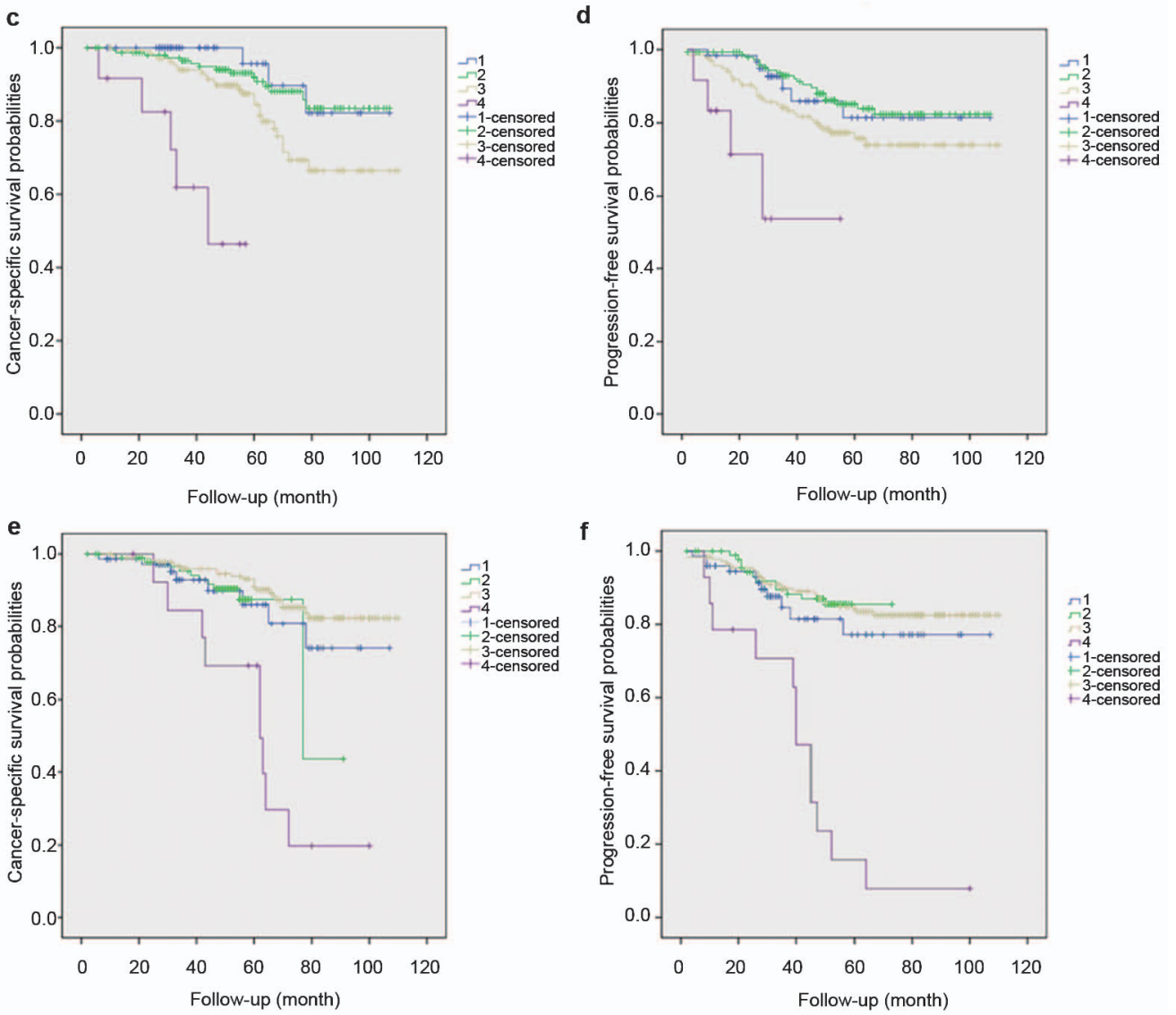

Figure 5 According to the 2010 staging system, the Kaplan-Meier curves did not show significant differences between the CSS and PFS rates for the T2b and T3a subgroups (a: $P=0.203$ for CSS; $\mathbf{b}: P=0.604$ for PFS). We divided the T3a group into two subgroups according to the diameter of the tumour (subgroup $1 \leqslant 10 \mathrm{~cm}$, subgroup2 $>10 \mathrm{~cm}$ ) and found that patients with subgroup2 tumours had poorer survival than T2b and subgroup1 patients (CSS rate $=0.040$ and 0.017 for subgroup2 vs. T2b and subgroup1, respectively, c; PFS rate $=0.222$ and 0.0478 for subgroup2 vs. T2b and subgroup1, respectively, d) (c, d: 1, T2b; 2, T3a subgroup1; 3, T3a subgroup2; 4, T3b). Finally, we divided the T3a group into three subgroups according to the extent of the tumour invasion (tumour invading the perirenal and/or renal sinus fat as subgroup 1 , tumour grossly extending into the renal vein as subgroup2, tumour invading the perirenal and/or renal sinus fat and renal vein as subgroup3) and found that patients with subgroup3 tumours had poorer survival than subgroup1 and subgroup2 patients (all CSS and PFS rates $<0.001$, e and f) (e, f: 1, T2b; 2, T3a subgroup1; 3, T3a subgroup2; 4, subgroup3). CSS, cancer-specific survival; PFS, progression-free survival.

staging system of RCC is suitable for worldwide application. Our present study showed that the revised TNM staging system of RCC effectively stratified the patient's risk of cancer progression and reflected the prognosis of our cohort of 1216 patients with ccRCC. Many studies have attempted to improve the accuracy of the TNM staging system, and previous studies have demonstrated that T3 tumours comprise a heterogeneous group of tumours that vary in prognosis. ${ }^{6}$ Many studies have found that tumours involving the direct ipsilateral adrenal gland can be recognized as predictive of a poorer prognosis, as those tumours act more aggressively than tumours involving the perirenal or renal sinus fat. These researchers have suggested that the direct ipsilateral adrenal gland involvement in 

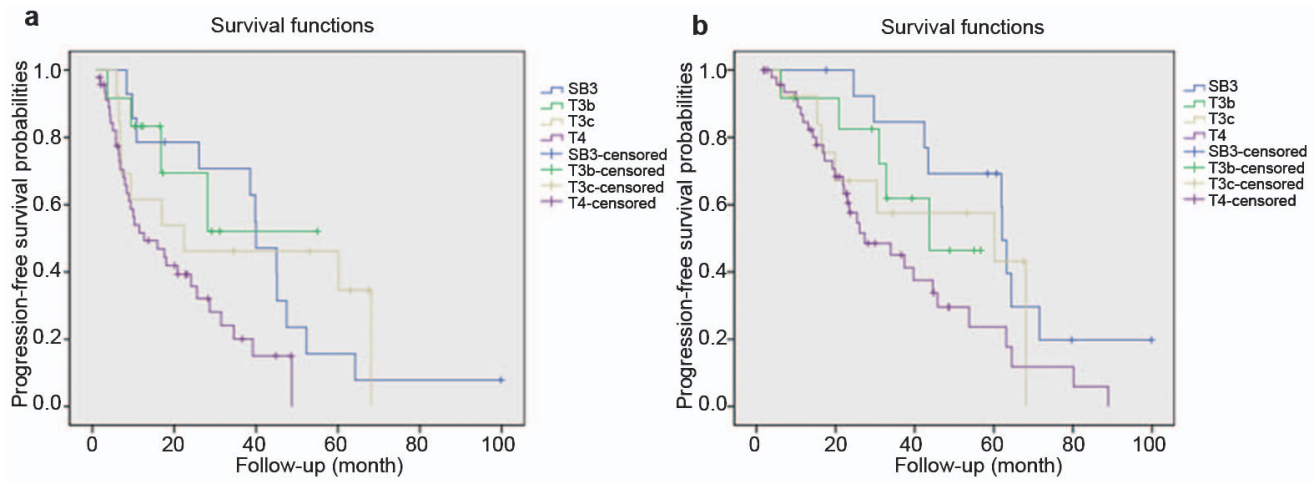

Figure 6 These Kaplan-Meier curves show that the PFS rates for patients with subgroup3 tumours demonstrated better survival than those with T3b, T3c or T4 (a: $P=0.016$ for PFS; $\mathbf{b}$ : $P=0.079$ for CSS) (SB3: subgroup3). CSS, cancer-specific survival; PFS, progression-free survival.

patients with pT3a in the old T staging system should be reclassified to pT4, as such tumours exhibit the same aggressive clinical behaviours as those extending beyond the Gerota's fascia. ${ }^{7-10}$ Therefore, in the revised 2010 TNM staging system, direct adrenal gland invasion was reclassified into the T4 stage. Our results were consistent with those of previous studies and supported this revision. In our study, 17 cases were upstaged from T3a to T4 according to the new staging system and demonstrated significantly poorer survival than the cases that remained in the T3a group. The improvements evidenced by this reclassification came about mainly as a consequence of the inclusion of adrenal invasion, a strong adverse prognostic factor, in the T4 disease category.

In previous studies, both Novara et al..$^{5}$ and Kim et al. ${ }^{11}$ showed that there was a significantly different survival rate between the T3a and T3b subgroups according to the revised 2010 staging system. Kaplan-Meier plots showed a significant difference in survival between the T3a and T3b subgroups (log-rank, $P=0.03$ ), indicating that the 2010 TNM staging system could accurately distinguish survival between T3a and T3b tumours. Moreover, 191 patients with T3b tumours were downstaged to T3a under the new staging system. Those patients who were shifted from T3b to T3a had significantly better prognoses than those who were not shifted.

However, we failed to demonstrate any significant difference in survival between the T2a and T2b subgroups. Our data were similar to those of Waalkes's study, ${ }^{12}$ which examined 579 patients with stage T2 RCC tumours. However, Novara et al. ${ }^{5}$ studied nearly 590 stage T2 RCC tumours and found significant a difference between the T2 subgroups. Therefore, further larger-scale studies should be conducted to confirm the significant difference between the outcomes of these two groups.

Furthermore, in our study, more than $92 \%$ of patients $(288 / 313)$ in the T3 stage were classified into the T3a subgroup. Veeratterapillay et al. ${ }^{13}$ found that $88 \%$ of their patients were classified into the T3a group, and they considered this to be a potential weakness of the revised TNM staging system, as the aim of any staging system providing prognostic data is to divide the patient population into groups bearing different outcomes. If almost all of the patients are divided into one group or subgroup, then the staging system could be perceived as having limited practical value. In addition, the survival of patients with T3a tumours was similar to the survival observed in T2b patients in our cohort. Thus, we considered whether it was necessary to divide the T3a group into different subgroups to predict the prognosis more accurately. We analysed the PFS and CSS with a cutoff of $7 \mathrm{~cm}$, but the results did not show any significant difference unless the cutoff was increased to $10 \mathrm{~cm}$. After dividing the T3a stage into two subgroups according to the diameter of the tumour (sub- group $1 \leqslant 10 \mathrm{~cm}$, subgroup $>10 \mathrm{~cm}$ ), we found that patients with subgroup2 tumours had poorer survival than T2b and subgroup1 patients. Gofrit et al. ${ }^{14}$ analysed the prognosis of patients with T3a tumours according to the 2002 staging system and found that tumour size was an important factor affecting outcomes in T3a tumours, a conclusion that was corroborated by our data. Then, we divided the T3a group into three subgroups, according to the extent of tumour invasion (tumour invading the perirenal and/or renal sinus fat as subgroup1, tumour grossly extending into the renal vein as subgroup2, and tumour invading the perirenal and/ or renal sinus fat and renal vein as subgroup 3 ), and found that patients with subgroup3 tumours had poorer survival than those in subgroup1 and subgroup2. These data suggest that more accurate classifications of T3a stages might more effectively predict the prognosis. Our sample size was relatively small and most likely of inadequate power to detect any significant changes in outcomes between patients with renal vein invasion and those with perirenal fat invasion. Novara et $a .^{5}$ studied the outcomes of 1059 patients with T3a tumours and found that there was no difference between the outcomes of patients with renal vein invasions and those with perinephric fat invasions. Our data were therefore in line with a larger study that supported the updated 2010 T3a classification.

\section{CONCLUSIONS}

To summarize, the revised 2010 TNM staging system better reflects the prognosis of patients with ccRCC in eastern China. However, some of the substages identified by the staging system have overlapping prognoses, and other substages include patients with heterogeneous outcomes. Further larger studies are needed to resolve these limitations in the T2 and T3a staging system. The staging system requires modifications, especially in the T3a staging system, to reduce the number of patients classified as T3a. In the present study, it was necessary to divide the T3a group into subgroups according to the tumour size $(\leqslant$ or $>10 \mathrm{~cm}$ ) or to the extent of invasion to more accurately predict the prognosis. This feasible modification should be validated by larger samples in the future.

\section{AUTHOR CONTRIBUTIONS}

$\mathrm{CQ}, \mathrm{QC}$ and JZ contributed to the analysis and interpretation of the data and drafted the manuscript. LJS, LC and PL helped to supply all of the patient data. GMZ, XM and PFS assisted with date analysis and helped to approve the final manuscript. MLW, ZDZ and MG participated in the collection of the data. CJY and WZ contributed to the conception and the design of the study. 


\section{COMPETING FINANCIAL INTERESTS}

The authors declare no competing financial interests.

\section{ACKNOWLEDGMENTS}

This work was supported by the Program for Development of Innovative Research Team of the First Affiliated Hospital of Nanjing Medical University, the Provincial Initiative Program for Excellency Disciplines, Jiangsu Province and the National Natural Science Foundation of China (NO. 81171963 and NO. 81201571).

1 Jemal A, Siegel R, Ward E, Hao Y, Xu J et al. Cancer statistics, 2009. CA Cancer J Clin 2009; 59: 225-49.

2 Yang L, Parkin DM, Ferlay J, Li L, Chen Y. Estimates of cancer incidence in China for 2000 and projections for 2005. Cancer Epidemiol Biomarkers Prev 2005; 14: 243-50.

3 Greene FL, Page DL, Fleming ID, Fritz A, Balch CM. AJCC Cancer Staging Manual. 6th ed. New York: Springer; 2002.

4 Greene FL, Gospodarowicz MK, Wittekend C. American Joint Committee on Cancer (AJCC) Staging Manual. 7th ed. Philadelphia, PA: Springer; 2009.

5 Novara G, Ficarra V, Antonelli A, Artibani W, Bertini R et al. Validation of the 2009 TNM version in a large multi-institutional cohort of patients treated for renal cell carcinoma: are further improvements needed? Eur Urol 2010; 58: 588-95.
6 Jung SJ, Ro JY, Truong LD, Ayala AG, Shen SS. Reappraisal of T3NO/NxMO renal cell carcinoma: significance of extent of fat invasion, renal vein invasion, and adrenal invasion. Hum Pathol 2008; 39: 1689-94.

7 Ficarra V, Galfano A, Guillé F, Schips L, Tostain J et al. A new staging system for locally advanced (pT3-4) renal cell carcinoma: a multicenter European study including 2,000 patients. J Urol 2007; 178: 418-24

8 Han KR, Bui MH, Pantuck AJ, Freitas DG, Leibovich BC et al. TNM T3a renal cell carcinoma: adrenal gland involvement is not the same as renal fat invasion. $J$ Urol 2003; 169: 899-904.

9 Thompson RH, Cheville JC, Lohse CM, Webster WS, Zincke H et al. Reclassification of patients with pT3 and pT4 renal cell carcinoma improves prognostic accuracy. Cancer 2005; 104: 53-60.

10 Fujita T, Iwamura M, Yanagisawa N, Muramoto M, Okayasu L et al. Reclassification of the current tumor, node, metastasis staging in $\mathrm{pT} 3$ renal cell carcinoma. Int J Urol 2008; 15: 582-6.

11 Kim SP, Alt AL, Weight CJ, Costello BA, Cheville JC et al. Independent validation of the 2010 American Joint Committee on Cancer TNM classification for renal cell carcinoma: results from a large, single institution cohort. J Urol 2011; 185: 2035-9.

12 Waalkes S, Becker F, Schrader AJ, Janssen M, Wegener G et al. Is there a need to further subclassify pT2 renal cell cancers as implemented by the revised 7th TNM version? Eur Urol 2011; 59: 258-63.

13 Veeratterapillay R, Simren R, El-Sherif A, Johnson MI, Soomro N et al. Accuracy of the revised 2010 TNM classification in predicting the prognosis of patients treated for renal cell cancer in the north east of England. J Clin Pathol 2012; 65: 367-71.

14 Gofrit ON, Shapiro A, Pizov G, Landau EH, Katz R et al. Does stage T3a renal cell carcinoma embrace a homogeneous group of patients? J Urol 2007; 177: 1682-6. 